\title{
An Introduction to ERP Systems: Architecture, Implementation and Impacts
}

\author{
Assma Habadi \\ Department of Electrical and \\ Computer Engineering, FOE, \\ King Abdulaziz University, \\ Jeddah, KSA
}

\author{
Yousra Samih \\ Department of Electrical and \\ Computer Engineering, FOE, \\ King Abdulaziz University, \\ Jeddah, KSA
}

\author{
Khadeejah Almehdar \\ Department of Electrical and \\ Computer Engineering, FOE, \\ King Abdulaziz University, \\ Jeddah, KSA
}

\author{
Eman Aljedani \\ Department of Electrical and Computer Engineering, FOE, \\ King Abdulaziz University, \\ Jeddah, KSA
}

\begin{abstract}
The Enterprise Resource Planning (ERP) is an integrated solution that have been revolutionizing the business processes in collaborative and distributed way. It considered as the backbone of the management systems due to the unlimited benefits it promises. However, many developing countries are not conscious of this impressive system. Therefore, this research paper explores and analyzes the ERP system architecture, implementation and, major effects when applying it into different areas, for enhancing replacing the traditional management systems with ERP systems in the developing countries.
\end{abstract}

\section{General Terms}

Management System, Interactive Database.

\section{Keywords}

ERP, traditional management, business processes, ERP architecture.

\section{INTRODUCTION}

Enterprise Resource Planning (ERP) is one of the most important breakthrough technologies that facilities the management system by providing many services. The ERP systems is a shared database that manage the organization's processes through supporting multiple functions and integrating several applications [1]. The worldwide organization's market for ERP services has increased of around $\$ 10$ billion in only four years [2]. This massive growing is due to the competitive advantages that organization's gain when integrating ERP to their management processes.

In the past decade, ERP system was widely spread to include many sectors. Since, the demand for handling the changing of administrations requirements, and the user satisfaction of having a convenient way to access, monitor, and deal with information is increasing. In addition, ERP systems allow the communication between different units that have various demands in a single system. This will peacefully permit all the end users to interact and utilize their resources using a single related system.

The paper is structured as follows: First, ERP various architectures is introduced in detail to select the architecture that is suitable for the organization's requirements. Implementation afterwards is pointed out to ensure full acknowledgment about ERP system from being just an idea until it becomes a complete system. Finally, ERP system effects is listed to declare the benefits of integrating it.

\section{ERP SYSTEMS ARCHITECTURE}

The architecture of the ERP system plays a major role to determine its success and durability for the institute. Today there are four major known architectures for the EPR systems [3][4][5]; each has its own advantages and weaknesses. The first is the Three-Tier architecture which is a scale up of the client/server Two-Tier architecture. It is composed of three layers Presentation, Application, and Database layer. Figure 1 shows a comparison of one, two, and three-tier architectures. As can be seen in the three-tier architecture the client is no longer directly communicating with the database, as a layer responsible for carrying out the business logic is introduced. In this architecture, the presentation layer is only responsible for browsing the data and providing a user-friendly interface, which allows the user to have less powerful machines. However, the application layer is where the data get retrieved and transferred to the database servers in the database layer. The application layer is also where the logic and the business rules are implemented.

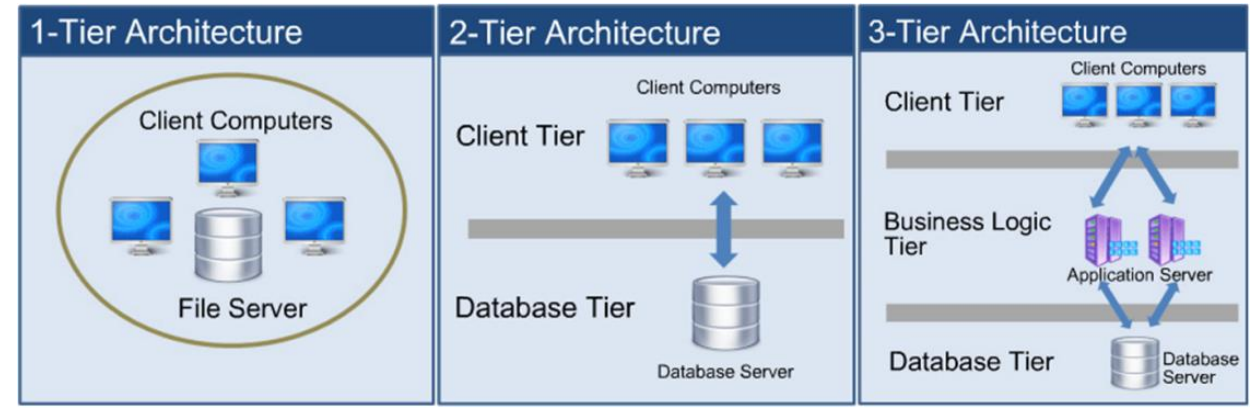

Fig 1: Different Tier Architecture 
Another architecture is the web-based; its mean goal is to allow remote users to access the ERP system. As can be seen in Figure 2, the web architecture has application and database layers same as the three-tier architecture. However, the presentation layer is split into two parts, the web services and web browser to support the mobility of the devices through the internet. Many institutes don't build their ERP as a web based architecture as the accessibility outside the organization may not be a requirement at first. But with the growth of the institute the remote access becomes a necessity, in which a web-enabled architecture is a solution. A web enabled architecture is an architecture that was not originally webbased but with additional new demands modification are made to make it web-enabled. This will surely limit the remote functionalities and capabilities.

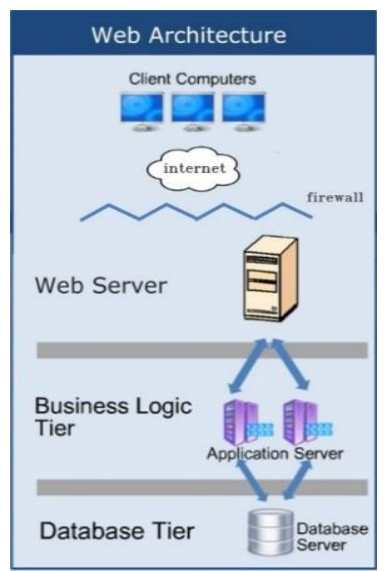

Fig 2: Web Architecture
SOA or service-oriented architecture is an approach used to create an architecture based upon the use of services, regardless of the networking methodologies used. With the growth of institutes the number of services it's required to deliver increase. With that a single system would not be able to keep up with the number of transactions. Therefore, a system for each service is lunched. SOA allows organizations to customize and modify their ERP system easily. In addition to that SOA encourages reusing existing services. It's worth mentioning that the communication across the different systems could be hard if regular interfaces are used, as they would be very expensive and intensive to develop; hence came the concept to standardize messages. In the IT landscape of an organization extensible markup language or XML is a way to standardize messages communicated between systems, as shown in Figure 3. XML is a way of describing information or data and is shed electronically disallows sharing of information in a consistent manner to support a concept of SOA. With XML, service oriented architecture becomes probable.

Even though cloud computing is becoming increasingly popular whether a well-established institute is willing to trust a third body to store and manage all of its information is still the question. New technologies demand for a new way of thinking and cloud computing is not the exception. As internet connections become faster and more reliable at least in the socalled first world, difference of access in terms of speed and conservation between local store and web-based data is decreasing considerably. As a result of access reliability this new technology has risen and is making its way into the technological arena. Figure 4 below shows a basic abstraction of the cloud computing architecture.

Unlike the three-tier and web based architectures; the SOA is not based on a certain technology or networking technique.

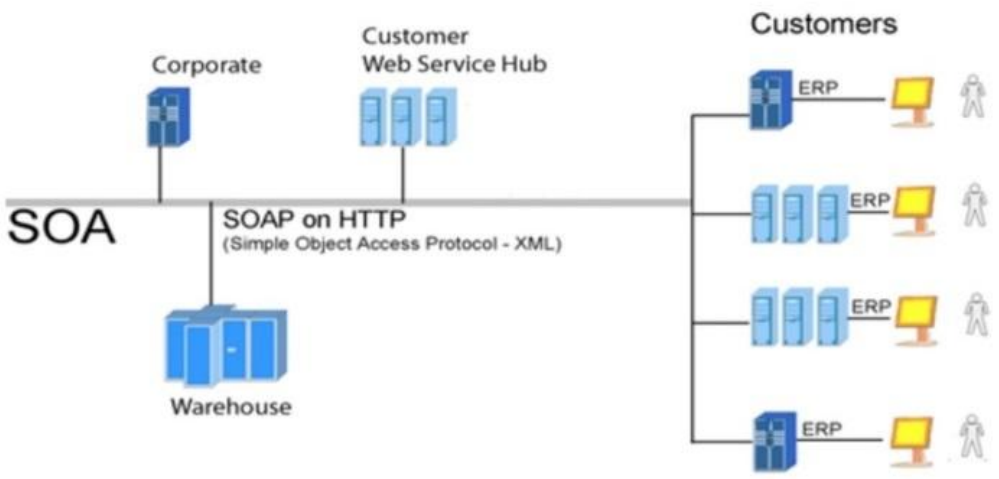

Fig 3: SOA Architecture

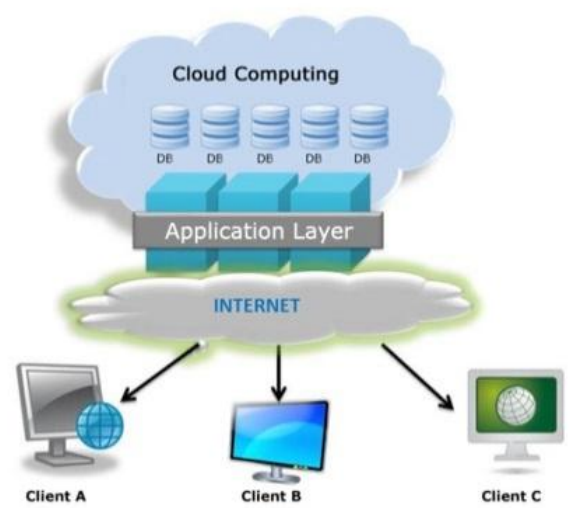

Fig 4: Cloud Computing Architecture 
Realizing the differences between the types of architectures can get confusing in this time and era. As it's every common to mix and match between them to get an architecture that this more suitable, reliable, and desirable for different institutions. The Venn diagram in Figure 5 can give clearer view of different architectures and their boundaries.

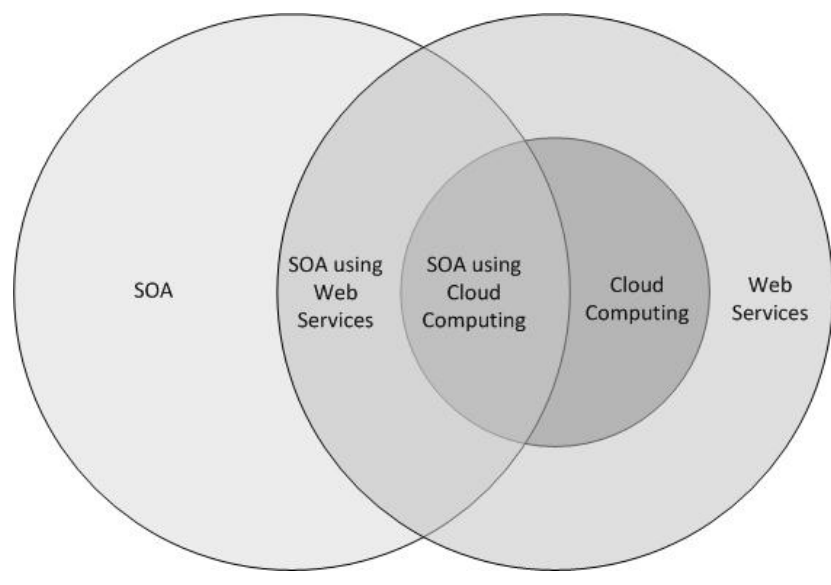

Fig 5: The Boundaries of Different Architectures

\section{CHOOSING SUITABLE ARCHITECTURE}

As each architecture has it pros and cons, choosing the right architecture for an institute can be tricky. First an institute has to specify the budget and limits in terms of employees responsible to operate and maintain the ERP system. Those limitations can determine if the architecture should be two or three-tier. The three-tier is surly scalable, reliable, and flexible, but it also can be costly and complex. However, in this day and age a reasonably sized institution should be able to afford it. As for the web-based architecture it main benefit is that it is runnable outside the walls of the organization. Yet the system might be slower in performance since the internet and more encryption is presented due to the data leaving the organization LAN. With that mentioned this will also affect the security of the data and may give a security bug for the hackers. This is also the case for the cloud computing architecture as its security lies in the hand of the vendors; causing organizations with more sensitive data to disregard it. On the other hand, if an organization was looking for a system that is expandable and independent, the SOA is the better choice, as it is more complex and advanced. It is also worth mentioning that the performance of each system will be much improved but the cross communication might be impacted.

\section{ERP SYSTEMS IMPLEMENTATION}

As stated in the introduction the demand for implementing ERP in organizations is increasing rapidly. Nowadays, around $40 \%$ of schools has already integrated ERP software [2] The strategy for implementing ERP has varied depending upon many vendors that offer different hosting services. Some of the leading vendors are: SAP, Oracle and Microsoft [6]. Although, they all follow similar basic methodology and pass through several phases which are: Preparation and Planning, Design, Development, Testing, Deployment, and Operation [7][8][9] in order to implement ERP into schools' curriculum. Each phase will be explained briefly as follows:

- Preparation and Planning
This step present the initiating phase for defining the project and sitting requirements. It includes issue identification, scope specification, project schedule and planning.

\section{- Design}

Here, the various configurations for prototypes are undertaken. This step helps defining the whole architecture and detailed requirements. Further, highlighting the design to be developed by the following phases.

\section{- Development}

The designs were specified earlier are developed in this phase. Multiple features and configurations will be added to finalize the required model for going live.

\section{- Testing}

In most cases this phase is overlapped with the development phase. It is responsible for systematic testing and gaps filling to satisfy the client needs.

\section{- Deployment}

In this phase, the installation will be configured at the customer sites. Hence, it defines as the prior step before going live, it also involves further system testing and support.

\section{- Operation}

This is the final step in which the ERP system is ready to go live and user start to use the system.

All these phases are presenting only the basic steps which are to be further divided into sub phases supported by many tools and frameworks which is out of the scope of this paper

However, the success of ERP system implementation is not guaranteed. There are between $67 \%$ and $90 \%$ of systems have failed and about 35\% of them have canceled [10]. Many studies and researches have been conducted regarding the success factors of ERP system implementation. But, they did not reach to an agreement about the main factors. Since, it may depend on the technology itself and the way it is implemented or from the end user perspective.

\section{ERP SYSTEMS EFFECTS}

As mentioned previously institutions have used ERP systems to cope with the changing environment and improve learning services. There are unlimited positive effects attracted the organization and institutions to start using ERP systems. Some of these benefits are summarized as follows [11] [12]:

- $\quad$ Reduce the access time to manage, analyze and process the data.

- Help organizing the data of institution.

- Avoid data redundancy and operations by using a common database.

- Increase efficiency and reduces paper use.

- Improve collaboration between department, staff, and costumers.

- Decrease expenses and save energy and time

- Streamline workflow and Systemize organizational processes.

- Provide high-quality services.

- Improve the internal communication and make it easy. 


\section{CONCLUSION}

Based on this research paper many findings have found. Firstly, generalizing the ERP system architecture is not possible as the requirements differ based on the institutions needs and the implementation it follows. Secondly, the ERP implementation has high failure rate therefore, the implementation processes must be applied wisely. Despite all of that, institutions have highly attracted to implement ERPs due to the dynamic grow of costumer's and the need for a flexible system that satisfy this expansion and suit the competitive environment. Finally, through this research paper it can be concluded that ERP technology will shape the future of management systems as it fits perfectly the demands of all organizations and institutions.

\section{REFERENCES}

[1] C. Chuang, "The Critical Success Factors for ERP Implementation in Higher Education," Proceedings of the 21st International Academic Conference, Miami, 2016.

[2] J. Willems and S. Bhuiyan, "Implementing Erp Software into Business School Curriculum: It Is More Common, Less Difficult and More Important Than You May Think," Issues in Information Systems, vol. 4, no. 1.

[3] S. Singhal, D. Puneet Tandon, and S. K. Sharma , "Hardware and Software Requirements for Implementation of ERP in Technical Education Institutes in India ," International Journal of Scientific \& Engineering Research, vol. 2, no. 7.

[4] M. and T., Enterprise Systems for Management Instructor's Manual.
[5] A. I. Gunawan and K. Surendro, "Enterprise architecture for cloud-based ERP system development," 2014 International Conference of Advanced Informatics: Concept, Theory and Application (ICAICTA), 2014.

[6] "Top 10 Enterprise Resource Planning (ERP) Vendors," compare business products.

[7] S. Nagpal, S. K. Khatri, and A. Kumar, "Comparative study of ERP implementation strategies," 2015 Long Island Systems, Applications and Technology, 2015.

[8] "6 Phases of an ERP Implementation Plan," 6 Phases of an ERP Implementation Plan | PC Bennett Solutions. [Online]. Available: http://pcbennett.com/blog/6-phaseserp-implementation-plan. [Accessed: 13-Apr-2017].

[9] S. Nagpal, S. K. Khatri, and P. K. Kapur, "Exploratory study to identify critical success factors penetration in ERP implementations," Proceedings of 3rd International Conference on Reliability, Infocom Technologies and Optimization, 2014.

[10] I. K. Sowan and R. Tahboub, "Erp Systems Critical Success Factors ICT Perspective," International Journal of Advanced Computer Science and Applications, vol. 6, no. 6 .

[11] D. Swartz and K. Orgill, "Higher Education ERP: lessons learned Using this framework for ERP could save your university millions of dollars," EDUCAUSE QUARTERLY.

[12] L. Zornada and T. Velkavrh, "Implementing ERP systems in higher education institutions," 27th International Conference on Information Technology Interfaces. 